\title{
Rhodium silyl hydrides in oxidation state +5 : classical or nonclassical?
}

\author{
Sergei F. Vyboishchikov and Georgii I. Nikonov
}

\section{Supporting materials}

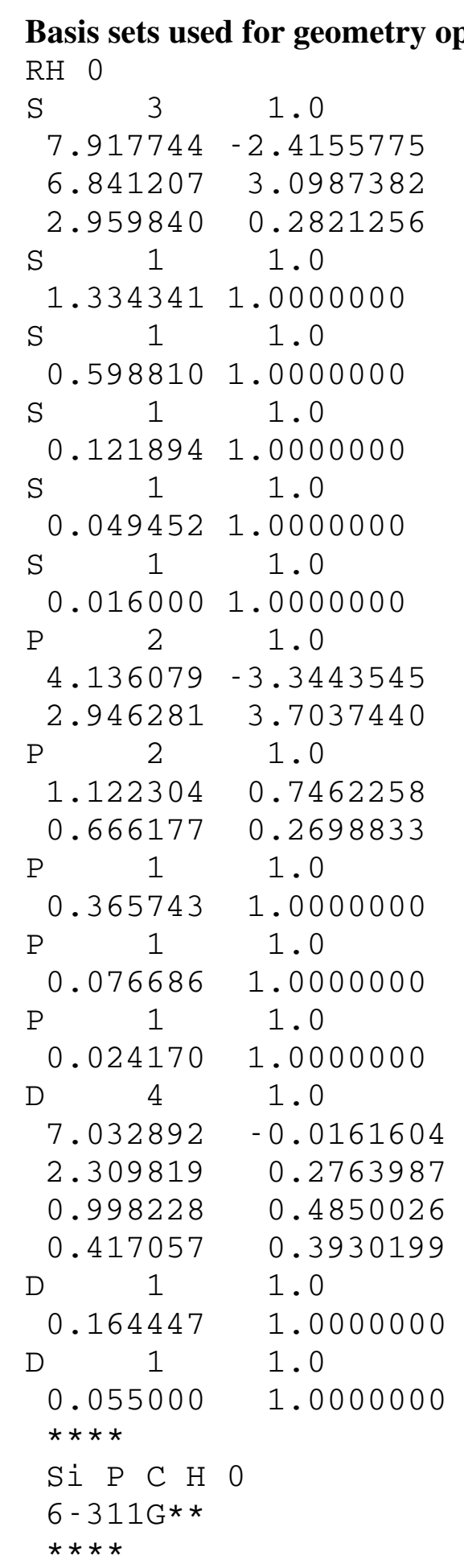


$\mathrm{RH} \quad 0$

RH- ECP 428

G POTENTIAL 1

21.00

S-G POTENTIAL 2

211.72

$2 \quad 5.82$

P-G POTENTIAL 2

210.42

$2 \quad 5.45$

D-G POTENTIAL 2

$2 \quad 8.82$

$2 \quad 3.87$

F-G POTENTIAL 2

212.31

26.16
0.000000

225.347754

32.823189

158.709412

26.444100

62.758626

10.978719

$-30.093456$

$-5.218482$ 


\section{Basis sets used for spin-spin coupling constant calculations (in Gaussian format)}

$\mathrm{RH} \quad 0$

$\begin{array}{lcc}\text { S } & 3 & 1.0 \\ 7.917744 & -2.4155775 \\ 6.841207 & 3.0987382 \\ 2.959840 & 0.2821256 \\ \text { S } & 1 & 1.0\end{array}$

1.3343411 .0000000

$\mathrm{S} \quad 1 \quad 1.0$

0.5988101 .0000000

S 11.0

0.1218941 .0000000

$\mathrm{S} \quad 1 \quad 1.0$

0.0494521 .0000000

$\mathrm{S} \quad 1 \quad 1.0$

0.0160001 .0000000

P 21.0

$4.136079-3.3443545$

$2.946281 \quad 3.7037440$

$\mathrm{P} \quad 2 \quad 1.0$

$\begin{array}{lll}1.122304 & 0.7462258\end{array}$

$0.666177 \quad 0.2698833$

$\mathrm{P} \quad 1 \quad 1.0$

0.3657431 .0000000

P 111.0

$0.076686 \quad 1.0000000$

P 111.0

$0.024170 \quad 1.0000000$

D $4 \quad 1.0$

$7.032892-0.0161604$

$2.309819 \quad 0.2763987$

$0.998228 \quad 0.4850026$

$0.417057 \quad 0.3930199$

D 111.0

$0.164447 \quad 1.0000000$

$\begin{array}{lcc}\mathrm{D} & 1 & 1.0 \\ 0.055000 & 1.0000000\end{array}$

$* * * *$

H 0 ! Hydride hydrogen

S 11.00

$136.32 \quad 1.0$

S 11.00

$68.16 \quad 1.0$

S 11.00

10.24651 .0

S 11.00

$2.34648 \quad 1.0$

S 11.00

0.673321 .0

S 11.00

0.224661 .0

S 11.00

0.0822171 .0 


$$
\begin{array}{ll}
\mathrm{P} \quad 1 & 1.00 \\
\mathrm{P} \quad 1 & 0.331 .0 \\
& 1.00 \\
& 1.301 .0
\end{array}
$$

Si 0

$$
\begin{aligned}
& \text { S } 11.00 \\
& 139978.6 \quad 1.0 \\
& \text { S } 11.00 \\
& 69989.3 \quad 1.0 \\
& \text { S } 11.00 \\
& 10380.21 .0 \\
& \text { S } 11.00 \\
& 2330.01 \quad 1.0 \\
& \text { S } 11.00 \\
& 657.466 \quad 1.0 \\
& \mathrm{~S} \quad 11.00 \\
& 214.004 \quad 1.0 \\
& \text { S } 11.00 \\
& 77.6064 \quad 1.0 \\
& \text { S } 11.00 \\
& 30.6395 \quad 1.0 \\
& \text { S } 11.00 \\
& 12.8156 \quad 1.0 \\
& \text { S } 11.00 \\
& 3.92714 \quad 1.0 \\
& \text { S } 11.00 \\
& 1.45221 \quad 1.0 \\
& \text { S } 11.00 \\
& 0.257644 \quad 1.0 \\
& \text { S } 11.00
\end{aligned}
$$$$
0.094404 \quad 1.0
$$$$
\text { P } 11.00
$$$$
401.6049 \quad 1.0
$$$$
\text { P } 11.00
$$$$
95.352426 \quad 1.0
$$$$
\text { P } 11.00
$$$$
30.334220 \quad 1.0
$$$$
\text { P } 11.00
$$$$
10.943727 \quad 1.0
$$$$
\text { P } 11.00
$$$$
4.0416822 \quad 1.0
$$$$
\mathrm{P} \quad 11.00
$$$$
1.4615479 \quad 1.0
$$

P 11.00

$$
0.33021241 \quad 1.0
$$

P 11.00

$$
0.09522172 \quad 1.0
$$

D 11.00

$$
0.15000000 \quad 1.0
$$
D 11.00

$0.60000000 \quad 1.0$

D 11.00 
**** $2.4000000 \quad 1.0$

C

$6-311 \mathrm{G} * *$

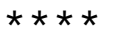

$\mathrm{H} 0$ ! non-hydride hydrogens $6-311 \mathrm{G} * *$

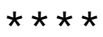

$\mathrm{RH} \quad 0$

$\mathrm{RH}-\mathrm{ECP} \quad 4 \quad 28$

G POTENTIAL

1

21.00

0.000000

S-G POTENTIAL 2

$2 \quad 11.72$

25.82

P-G POTENTIAL

2

$2 \quad 10.42$

$2 \quad 5.45$

D-G POTENTIAL

2

$2 \quad 8.82$

$2 \quad 3.87$

8626

F-G POTENTIAL

225.347754

32.823189

158.709412

26.444100

2

$2 \quad 12.31$

$-30.093456$

26.16

$-5.218482$ 


\section{Complex 1 - $\mathrm{CpRh}\left(\mathrm{SiMe}_{3}\right)_{2} \mathrm{H}_{2}$}

$\mathrm{Rh}$

$\mathrm{H}$

C

C

C

C

C

$\mathrm{H}$

$\mathrm{H}$

$\mathrm{H}$

$\mathrm{H}$

$\mathrm{H}$

$\mathrm{H}$

$\mathrm{Si}$

$\mathrm{Si}$

C

$\mathrm{H}$

$\mathrm{H}$

$\mathrm{H}$

C

$\mathrm{H}$

$\mathrm{H}$

$\mathrm{H}$

C

$\mathrm{H}$

$\mathrm{H}$

$\mathrm{H}$

C

$\mathrm{H}$

$\mathrm{H}$

$\mathrm{H}$

C

$\mathrm{H}$

$\mathrm{H}$

$\mathrm{H}$

C

$\mathrm{H}$

$\mathrm{H}$

$\mathrm{H}$
- 0.000731

0.002055

1. 097214

$-0.127503$

$-1.211655$

$-0.660002$

0.770564

2.097958

$-0.218191$

$-2.269804$

$-1.224502$

1.477210

0.006661

2. 000480

$-1.982410$

2. 892398

3. 098464

3. 856311

2. 289911

1.806367

1. 348471

1.192313

2. 803302

3.193693

3.491661

2. 738762

4.111185

$-1.752062$

$-1.176627$

$-1.236808$

$-2.742513$

$-2.946358$

$-2.363421$

$-3.887829$

$-3.118534$

$-3.399832$

$-4.047562$

$-2.629361$
$-3.202630$
$-0.386314$

0.716667

$-2.354507$

$-2.338491$

$-2.339372$

$-2.332803$

$-2.343948$

$-2.389205$

$-2.345057$

$-2.355955$

$-2.343503$

$-2.369705$

0.633209

0.933231

0.960696

0.768539

$-0.282416$

1.305356

1.196252

2. 794744

3. 002445

3. 286413

3. 267265

0.281291

$-0.761998$

0.328791

0.894794

2.836881

3. 259790

3.108984

3. 322974

0.692347

$-0.366138$

1.030142

1. 267952

0.420907

$-0.641050$

1.016756

0.572859
$-0.022487$

1.106341

0.486265

1. 227870

0.293808

$-1.027798$

$-0.909661$

0.910720

2. 311622

0.544432

$-1.957295$

$-1.735288$

$-1.219595$

0.003500

0.002442

1. 680079

1.934659

1.651504

2. 496540

$-0.315145$

$-1.294178$

0.453807

$-0.301894$

$-1.333725$

$-1.145748$

$-2.335063$

$-1.346680$

$-0.170066$

0.667259

$-1.103326$

$-0.175995$

1.625300

1. 785792

2.496184

1. 603418

$-1.430162$

$-1.356118$

$-1.418327$

$-2.404656$

\section{Complex 2 - CpRh( $\left(\mathrm{SiMe}_{3}\right)_{3} \mathrm{H}$}

$\mathrm{Rh}$

$\mathrm{H}$

C

C

C

C

C

$\mathrm{H}$

$\mathrm{H}$
0.004812

$-0.055045$

1.111203

$-0.166140$

$-1.159973$

$-0.498309$

0.916616

2.071061

$-0.353489$
$-0.418954$

$-0.508822$

$-2.055744$

$-2.605329$

$-1.764223$

$-0.698051$

$-0.869494$

$-2.476817$

$-3.499302$
$-0.339199$

1. 239212

$-1.589193$

$-1.279121$

$-1.873997$

$-2.557249$

$-2.376060$

$-1.300273$

$-0.688740$ 


\begin{tabular}{|c|c|c|c|}
\hline $\mathrm{H}$ & -2.235792 & -1.919756 & -1.837541 \\
\hline $\mathrm{H}$ & -0.980571 & 0.077045 & -3.145201 \\
\hline $\mathrm{H}$ & 1.698821 & -0.251810 & -2.810536 \\
\hline $\mathrm{Si}$ & 0.000493 & 1.982951 & -0.531956 \\
\hline $\mathrm{Si}$ & 1.999920 & -0.305135 & 1.056844 \\
\hline $\mathrm{Si}$ & -2.029068 & -0.317591 & 1.019727 \\
\hline $\mathrm{C}$ & 2.160853 & -1.972143 & 1.975256 \\
\hline $\mathrm{H}$ & 2.122958 & -2.827822 & 1.283455 \\
\hline $\mathrm{H}$ & 3.123302 & -2.013833 & 2.513394 \\
\hline $\mathrm{H}$ & 1.356008 & -2.108694 & 2.714839 \\
\hline C & 2.088849 & 1.023513 & 2.415126 \\
\hline $\mathrm{H}$ & 2.149525 & 2.045945 & 2.015419 \\
\hline $\mathrm{H}$ & 1.223217 & 0.975030 & 3.093269 \\
\hline $\mathrm{H}$ & 2.997315 & 0.846153 & 3.016091 \\
\hline C & 3.594065 & -0.139552 & 0.021083 \\
\hline $\mathrm{H}$ & 3.747174 & -1.006032 & -0.639515 \\
\hline $\mathrm{H}$ & 3.613394 & 0.768281 & -0.596328 \\
\hline $\mathrm{H}$ & 4.452551 & -0.095995 & 0.713814 \\
\hline $\mathrm{C}$ & 1.700302 & 2.716324 & -1.026620 \\
\hline $\mathrm{H}$ & 2.129004 & 2.207486 & -1.904155 \\
\hline $\mathrm{H}$ & 1.554019 & 3.777011 & -1.295428 \\
\hline $\mathrm{H}$ & 2.444446 & 2.679145 & -0.217530 \\
\hline C & -0.569995 & 3.076980 & 0.927330 \\
\hline $\mathrm{H}$ & -0.022592 & 2.887401 & 1.860866 \\
\hline $\mathrm{H}$ & -0.387545 & 4.127314 & 0.640482 \\
\hline $\mathrm{H}$ & -1.645880 & 2.976905 & 1.133729 \\
\hline C & -1.121597 & 2.555537 & -1.979854 \\
\hline $\mathrm{H}$ & -0.681833 & 2.300289 & -2.956871 \\
\hline $\mathrm{H}$ & -2.139331 & 2.140743 & -1.941342 \\
\hline $\mathrm{H}$ & -1.206409 & 3.655739 & -1.940978 \\
\hline C & -1.926023 & 0.394675 & 2.783959 \\
\hline $\mathrm{H}$ & -1.268975 & -0.221772 & 3.418828 \\
\hline $\mathrm{H}$ & -1.559198 & 1.428704 & 2.819950 \\
\hline $\mathrm{H}$ & -2.932569 & 0.376538 & 3.235630 \\
\hline $\mathrm{C}$ & -2.593980 & -2.119664 & 1.294852 \\
\hline $\mathrm{H}$ & -2.880651 & -2.624849 & 0.36083 \\
\hline $\mathrm{H}$ & -1.805716 & -2.720215 & 1.774841 \\
\hline $\mathrm{H}$ & -3.472583 & -2.123420 & 1.962719 \\
\hline C & -3.462038 & 0.572623 & 0.135025 \\
\hline $\mathrm{H}$ & -3.614094 & 0.191630 & -0.88623 \\
\hline $\mathrm{H}$ & -4.389381 & 0.395251 & 0.70708 \\
\hline $\mathrm{H}$ & -3.309482 & 1.659588 & 0.0723 \\
\hline
\end{tabular}

$\begin{array}{lccr}\text { Complex } 2-\mathbf{C p R h}\left(\mathrm{SiMe}_{3}\right)_{3} \mathrm{H} \text { calculated by the ONIOM method } \\ \text { Rh } & 0.004554 & -0.362929 & -0.341399 \\ \mathrm{H} & -0.008640 & -0.371834 & 1.235564 \\ \mathrm{C} & 1.082659 & -2.070958 & -1.519060 \\ \mathrm{C} & -0.229600 & -2.543832 & -1.247853 \\ \mathrm{C} & -1.156059 & -1.656615 & -1.890462 \\ \mathrm{C} & -0.409355 & -0.646023 & -2.571727 \\ \mathrm{C} & 0.984463 & -0.884518 & -2.327385 \\ \mathrm{H} & 2.003225 & -2.555575 & -1.203849\end{array}$




$\begin{array}{lrrr}\mathrm{H} & -0.481945 & -3.422560 & -0.659310 \\ \mathrm{H} & -2.238047 & -1.764338 & -1.915184 \\ \mathrm{H} & -0.825367 & 0.112618 & -3.226232 \\ \mathrm{H} & 1.815315 & -0.319132 & -2.741404 \\ \mathrm{Si} & -0.057083 & 2.054879 & -0.610019 \\ \mathrm{Si} & 2.018826 & -0.339974 & 1.055680 \\ \mathrm{Si} & -2.023795 & -0.409549 & 1.065247 \\ \mathrm{C} & 2.109374 & -1.990623 & 1.978111 \\ \mathrm{H} & 2.110595 & -2.828210 & 1.252176 \\ \mathrm{H} & 3.039347 & -2.025249 & 2.581736 \\ \mathrm{H} & 1.230961 & -2.087965 & 2.648722 \\ \mathrm{C} & 2.107957 & 1.013088 & 2.372820 \\ \mathrm{H} & 2.235357 & 2.003915 & 1.896344 \\ \mathrm{H} & 1.185924 & 0.999610 & 2.988179 \\ \mathrm{H} & 2.981095 & 0.820537 & 3.029100 \\ \mathrm{C} & 3.582991 & -0.208648 & -0.005046 \\ \mathrm{H} & 3.766167 & -1.163145 & -0.535996 \\ \mathrm{H} & 3.478852 & 0.603806 & -0.748112 \\ \mathrm{H} & 4.450827 & 0.005087 & 0.651777 \\ \mathrm{C} & 1.617778 & 2.724345 & -1.185678 \\ \mathrm{H} & 1.973427 & 2.136056 & -2.055346 \\ \mathrm{H} & 1.500545 & 3.785123 & -1.487978 \\ \mathrm{H} & 2.356394 & 2.668123 & -0.363541 \\ \mathrm{C} & -0.560425 & 3.069937 & 0.905759 \\ \mathrm{H} & 0.005189 & 2.751653 & 1.798701 \\ \mathrm{H} & -0.344476 & 4.139859 & 0.708778 \\ \mathrm{H} & -1.646533 & 2.960875 & 1.089480 \\ \mathrm{C} & -1.281982 & 2.539205 & -1.973019 \\ \mathrm{H} & -0.793856 & 2.444201 & -2.963944 \\ \mathrm{H} & -2.174982 & 1.885848 & -1.935682 \\ \mathrm{H} & -1.591707 & 3.594664 & -1.830694 \\ \mathrm{C} & -1.880217 & 0.285986 & 2.826028 \\ \mathrm{H} & -1.092549 & -0.262186 & 3.382933 \\ \mathrm{H} & -1.637441 & 1.362659 & 2.811157 \\ \mathrm{H} & -2.849221 & 0.147535 & 3.347676 \\ \mathrm{C} & -2.500391 & -2.225041 & 1.294151 \\ \mathrm{H} & -2.811781 & -2.662811 & 0.325079 \\ \mathrm{H} & -1.630461 & -2.784745 & 1.694902 \\ \mathrm{H} & -3.344900 & -2.295570 & 2.009853 \\ \mathrm{C} & -3.479261 & 0.449354 & 0.217143 \\ \mathrm{H} & -3.589490 & 0.062920 & -0.816092 \\ \mathrm{H} & -4.407611 & 0.239454 & 0.786736 \\ & -3.314198 & 1.543597 & 0.190158\end{array}$

\section{Complex 2' - $\mathrm{CpRh}\left(\mathrm{SiH}_{3}\right)_{3} \mathrm{H}$}

$\begin{array}{lr}\text { Rh } & -0.069392 \\ \mathrm{H} & 0.673381 \\ \mathrm{C} & -2.042688 \\ \mathrm{C} & -2.284923 \\ \mathrm{C} & -2.062110 \\ \mathrm{C} & -1.677291 \\ \mathrm{C} & -1.663888\end{array}$

$\begin{array}{rr}-0.004851 & -0.168114 \\ -0.007424 & -1.569024 \\ 1.155449 & 0.035746 \\ 0.068720 & -0.863313 \\ -1.154005 & -0.153965 \\ -0.826965 & 1.189402 \\ 0.606496 & 1.307665\end{array}$




$\begin{array}{lrrr}\mathrm{H} & -2.145318 & 2.213189 & -0.196690 \\ \mathrm{H} & -2.581895 & 0.157596 & -1.905842 \\ \mathrm{H} & -2.172527 & -2.157010 & -0.560172 \\ \mathrm{H} & -1.452655 & -1.535178 & 1.983536 \\ \mathrm{H} & -1.444419 & 1.172889 & 2.209891 \\ \mathrm{Si} & 1.676161 & 0.029307 & 1.463089 \\ \mathrm{H} & 1.969343 & 1.413021 & 1.986454 \\ \mathrm{H} & 2.999151 & -0.537420 & 1.027279 \\ \mathrm{H} & 1.230190 & -0.778960 & 2.654269 \\ \mathrm{Si} & 1.112805 & 1.978608 & -0.787172 \\ \mathrm{H} & 0.683481 & 2.463327 & -2.147744 \\ \mathrm{H} & 2.607661 & 1.840418 & -0.833091 \\ \mathrm{H} & 0.790223 & 3.090906 & 0.173722 \\ \mathrm{Si} & 1.207370 & -1.934356 & -0.786673 \\ \mathrm{H} & 2.556259 & -1.666995 & -1.383547 \\ \mathrm{H} & 0.429598 & -2.725592 & -1.806778 \\ \mathrm{H} & 1.416878 & -2.852459 & 0.390255\end{array}$

\section{Complex 3a - CpRh $\left(\mathrm{SiMe}_{3}\right)_{2}\left(\mathrm{SiEt}_{3}\right) \mathrm{H}$}

\begin{tabular}{|c|c|c|c|}
\hline $\mathrm{Rh}$ & -0.346674 & -0.185972 & -0.556545 \\
\hline $\mathrm{H}$ & 0.079402 & 1.177259 & 0.130088 \\
\hline $\mathrm{C}$ & -1.377936 & -0.204383 & -2.649877 \\
\hline C & 0.011994 & -0.052315 & -2.920441 \\
\hline C & 0.683734 & -1.213039 & -2.422105 \\
\hline $\mathrm{C}$ & -0.288988 & -2.086192 & -1.845804 \\
\hline C & -1.575149 & -1.458642 & -1.975252 \\
\hline $\mathrm{H}$ & -2.160577 & 0.494958 & -2.932957 \\
\hline $\mathrm{H}$ & 0.479373 & 0.795833 & -3.415358 \\
\hline $\mathrm{H}$ & 1.748439 & -1.417377 & -2.497084 \\
\hline $\mathrm{H}$ & -0.094697 & -3.068501 & -1.425160 \\
\hline $\mathrm{H}$ & -2.530679 & -1.883232 & -1.677720 \\
\hline $\mathrm{Si}$ & -0.893857 & -1.416796 & 1.440594 \\
\hline $\mathrm{Si}$ & -2.020278 & 1.488352 & 0.037153 \\
\hline $\mathrm{Si}$ & 1.877714 & 0.339713 & 0.376142 \\
\hline C & -1.689902 & 3.063389 & -0.992273 \\
\hline $\mathrm{H}$ & -1.686544 & 2.857334 & -2.074071 \\
\hline $\mathrm{H}$ & -2.483138 & 3.804783 & -0.794201 \\
\hline $\mathrm{H}$ & -0.724283 & 3.529276 & -0.741908 \\
\hline C & -2.130255 & 2.072129 & 1.845438 \\
\hline $\mathrm{H}$ & -2.495312 & 1.285582 & 2.521243 \\
\hline $\mathrm{H}$ & -1.165714 & 2.431852 & 2.233751 \\
\hline $\mathrm{H}$ & -2.845657 & 2.911102 & 1.89432 \\
\hline $\mathrm{C}$ & -3.789769 & 0.949074 & -0.439724 \\
\hline $\mathrm{H}$ & -3.903154 & 0.791353 & -1.522726 \\
\hline $\mathrm{H}$ & -4.115045 & 0.033589 & 0.072426 \\
\hline $\mathrm{H}$ & -4.481583 & 1.759786 & -0.150973 \\
\hline C & -2.770554 & -1.709545 & 1.695403 \\
\hline $\mathrm{H}$ & -3.257244 & -2.100510 & 0.788286 \\
\hline $\mathrm{H}$ & -2.889655 & -2.467289 & 2.48959 \\
\hline $\mathrm{H}$ & -3.316835 & -0.809847 & 2.01300 \\
\hline C & -0.291820 & -0.807856 & 3.14945 \\
\hline $\mathrm{H}$ & -0.614402 & 0.215209 & 3.38963 \\
\hline
\end{tabular}




$\begin{array}{rrrr}\mathrm{H} & -0.726196 & -1.480049 & 3.910154 \\ \mathrm{H} & 0.801664 & -0.856381 & 3.257676 \\ \mathrm{C} & -0.233436 & -3.214412 & 1.333357 \\ \mathrm{H} & -0.811512 & -3.810662 & 0.609355 \\ \mathrm{H} & 0.828346 & -3.277241 & 1.058006 \\ \mathrm{H} & -0.355003 & -3.693951 & 2.320221 \\ \mathrm{C} & 2.868693 & -1.199948 & 0.964969 \\ \mathrm{H} & 3.783783 & -0.778981 & 1.423278 \\ \mathrm{H} & 2.325421 & -1.683986 & 1.792632 \\ \mathrm{C} & 1.996883 & 1.584786 & 1.836375 \\ \mathrm{H} & 1.316761 & 1.265870 & 2.641127 \\ \mathrm{H} & 3.015545 & 1.473142 & 2.252224 \\ \mathrm{C} & 2.882771 & 1.147869 & -1.051408 \\ \mathrm{H} & 2.350774 & 2.063951 & -1.363584 \\ \mathrm{H} & 2.866007 & 0.486964 & -1.933013 \\ \mathrm{C} & 4.340663 & 1.481849 & -0.684207 \\ \mathrm{H} & 4.867957 & 1.962922 & -1.524851 \\ \mathrm{H} & 4.403109 & 2.169567 & 0.174220 \\ \mathrm{H} & 4.910884 & 0.577292 & -0.417948 \\ \mathrm{C} & 1.745201 & 3.062563 & 1.494549 \\ \mathrm{H} & 0.740125 & 3.222401 & 1.071882 \\ \mathrm{H} & 1.823002 & 3.703512 & 2.388276 \\ \mathrm{H} & 2.468688 & 3.443309 & 0.756394 \\ \mathrm{C} & 3.260802 & -2.241887 & -0.091531 \\ \mathrm{H} & 3.887018 & -3.039405 & 0.341548 \\ \mathrm{H} & 2.376488 & -2.727454 & -0.532476 \\ \mathrm{H} & 3.837080 & -1.791629 & -0.916758\end{array}$

\section{Complex 3a' $-\mathrm{CpRh}\left(\mathrm{SiMe}_{3}\right)_{2}\left(\mathrm{SiEt}_{3}\right) \mathrm{H}$}

\section{$\mathrm{Rh}$}

$\mathrm{H}$

C

C

C

C

C

$\mathrm{H}$

$\mathrm{H}$

$\mathrm{H}$

$\mathrm{H}$

$\mathrm{H}$

Si

Si

Si

C

$\mathrm{H}$

$\mathrm{H}$

$\mathrm{H}$

C

$\mathrm{H}$

$\mathrm{H}$

$\mathrm{H}$
0.482774

$-0.017451$

1.749398

0.393194

$-0.285132$

0.653638

1.920741

2.530238

$-0.050224$

$-1.326743$

0.451471

2.854297

0.730230

2.032320

$-1.841562$

1.792321

1.880559

2. 555750

0.802505

1.895357

2.123657

0.897002

2.626206
$-0.507757$

$-0.103977$

$-2.450386$

$-2.882494$

$-2.234351$

$-1.407032$

$-1.529708$

$-2.784624$

$-3.577690$

$-2.373095$

$-0.823699$

$-1.062147$

1.739160

$-0.030879$

0.013127

$-1.375305$

$-2.391712$

$-1.262643$

$-1.297416$

1.619068

2.492277

1.764706

1.603246
$-0.245174$

1.200829

$-0.596069$

$-0.579277$

$-1.659046$

$-2.350189$

$-1.686361$

0.082748

0.129797

$-1.935826$

$-3.243855$

$-1.989591$

$-1.074902$

1. 575350

0.393819

2. 910015

2. 496718

3. 698988

3.387308

2. 511937

1. 884303

2.951937

3.338822 


$\begin{array}{lrrr}\mathrm{C} & 3.852786 & -0.162553 & 1.018956 \\ \mathrm{H} & 4.106445 & -1.162039 & 0.636031 \\ \mathrm{H} & 4.111367 & 0.573826 & 0.246118 \\ \mathrm{H} & 4.495584 & 0.028739 & 1.896017 \\ \mathrm{C} & 2.556276 & 2.281947 & -1.275876 \\ \mathrm{H} & 3.149600 & 1.531457 & -1.821230 \\ \mathrm{H} & 2.579484 & 3.216403 & -1.863601 \\ \mathrm{H} & 3.058926 & 2.482434 & -0.318673 \\ \mathrm{C} & -0.094729 & 3.197636 & -0.154957 \\ \mathrm{H} & 0.263046 & 3.322746 & 0.877031 \\ \mathrm{H} & 0.156047 & 4.120157 & -0.707404 \\ \mathrm{H} & -1.192027 & 3.122369 & -0.125426 \\ \mathrm{C} & 0.070679 & 1.896519 & -2.869449 \\ \mathrm{H} & 0.712116 & 1.352198 & -3.580552 \\ \mathrm{H} & -0.961059 & 1.540224 & -2.999179 \\ \mathrm{H} & 0.097838 & 2.961526 & -3.159069 \\ \mathrm{C} & -2.940232 & 0.720872 & -1.015149 \\ \mathrm{H} & -3.909520 & 0.930108 & -0.524391 \\ \mathrm{H} & -2.550671 & 1.706432 & -1.319417 \\ \mathrm{C} & -2.059605 & 1.176476 & 1.917014 \\ \mathrm{H} & -1.948139 & 0.528163 & 2.807150 \\ \mathrm{H} & -1.210233 & 1.875725 & 1.955755 \\ \mathrm{C} & -2.602504 & -1.675671 & 0.917666 \\ \mathrm{H} & -1.950705 & -2.101376 & 1.701385 \\ \mathrm{H} & -2.534883 & -2.375758 & 0.068099 \\ \mathrm{C} & -4.054635 & -1.612215 & 1.422240 \\ \mathrm{H} & -4.430166 & -2.613158 & 1.693417 \\ \mathrm{H} & -4.147256 & -0.978089 & 2.318150 \\ \mathrm{H} & -4.737059 & -1.205243 & 0.658219 \\ \mathrm{C} & -3.369553 & 1.977605 & 2.015583 \\ \mathrm{H} & -4.260563 & 1.330490 & 1.999727 \\ \mathrm{H} & -3.407382 & 2.564085 & 2.948562 \\ \mathrm{H} & -3.469478 & 2.689739 & 1.181061 \\ \mathrm{C} & -3.181391 & -0.158521 & -2.248463 \\ \mathrm{H} & -3.836913 & 0.344854 & -2.978279 \\ \mathrm{H} & -2.242249 & -0.400122 & -2.769560 \\ \mathrm{H} & -3.667722 & -1.111094 & -1.980461\end{array}$

\section{Complex 3b - CpRh(SiMe $)_{2}\left(\operatorname{SiEt}_{3}\right) \mathbf{H}$}

$\begin{array}{llrr}\mathrm{Rh} & -0.617632 & -0.080359 & -0.444789 \\ \mathrm{H} & -1.142443 & 0.371934 & 0.980804 \\ \mathrm{C} & -1.639058 & -1.569445 & -1.940484 \\ \mathrm{C} & -2.406152 & -0.370354 & -2.021045 \\ \mathrm{C} & -1.540783 & 0.667397 & -2.486001 \\ \mathrm{C} & -0.241236 & 0.114945 & -2.703363 \\ \mathrm{C} & -0.296247 & -1.279257 & -2.356450 \\ \mathrm{H} & -2.014164 & -2.545691 & -1.642714 \\ \mathrm{H} & -3.457998 & -0.261617 & -1.767040 \\ \mathrm{H} & -1.827354 & 1.698567 & -2.677470 \\ \mathrm{H} & 0.619151 & 0.645821 & -3.101306 \\ \mathrm{H} & 0.508906 & -1.999488 & -2.470417 \\ \mathrm{Si} & 1.724420 & -0.145069 & 0.192383\end{array}$




\begin{tabular}{|c|c|c|c|}
\hline $\mathrm{Si}$ & -1.181093 & -1.640238 & 1.347808 \\
\hline $\mathrm{Si}$ & -0.955024 & 2.219961 & 0.332602 \\
\hline C & -3.077841 & -1.565097 & 1.556484 \\
\hline $\mathrm{H}$ & -3.600420 & -1.784903 & 0.612372 \\
\hline $\mathrm{H}$ & -3.402578 & -2.309614 & 2.303603 \\
\hline $\mathrm{H}$ & -3.414907 & -0.574033 & 1.898898 \\
\hline $\mathrm{C}$ & -0.453059 & -1.316496 & 3.075572 \\
\hline $\mathrm{H}$ & 0.641915 & -1.417118 & 3.099051 \\
\hline $\mathrm{H}$ & -0.705844 & -0.313308 & 3.452266 \\
\hline $\mathrm{H}$ & -0.871240 & -2.055821 & 3.780061 \\
\hline $\mathrm{C}$ & -0.801640 & -3.469356 & 0.964884 \\
\hline $\mathrm{H}$ & -1.251182 & -3.813157 & 0.021906 \\
\hline $\mathrm{H}$ & 0.273093 & -3.690419 & 0.930200 \\
\hline $\mathrm{H}$ & -1.240027 & -4.071603 & 1.780169 \\
\hline C & 2.447300 & -1.913661 & 0.506544 \\
\hline $\mathrm{H}$ & 3.483847 & -1.712347 & 0.837290 \\
\hline $\mathrm{H}$ & 1.954312 & -2.368818 & 1.382495 \\
\hline $\mathrm{C}$ & 2.331145 & 0.784645 & 1.780326 \\
\hline $\mathrm{H}$ & 3.186233 & 0.183017 & 2.141621 \\
\hline $\mathrm{H}$ & 1.569426 & 0.689378 & 2.571347 \\
\hline $\mathrm{C}$ & 2.812884 & 0.519106 & -1.262237 \\
\hline $\mathrm{H}$ & 2.529004 & -0.014981 & -2.184909 \\
\hline $\mathrm{H}$ & 2.553644 & 1.576808 & -1.444271 \\
\hline $\mathrm{C}$ & -0.743903 & 2.642030 & 2.179426 \\
\hline $\mathrm{H}$ & -1.536539 & 2.151946 & 2.768635 \\
\hline $\mathrm{H}$ & 0.221673 & 2.356256 & 2.613916 \\
\hline $\mathrm{H}$ & -0.865255 & 3.730971 & 2.311167 \\
\hline $\mathrm{C}$ & -2.793156 & 2.648057 & 0.039702 \\
\hline $\mathrm{H}$ & -3.093783 & 2.604270 & -1.017173 \\
\hline $\mathrm{H}$ & -3.451836 & 1.967985 & 0.602712 \\
\hline $\mathrm{H}$ & -2.986375 & 3.672947 & 0.401245 \\
\hline $\mathrm{C}$ & 0.065546 & 3.447165 & -0.707402 \\
\hline $\mathrm{H}$ & -0.190247 & 3.367408 & -1.774909 \\
\hline $\mathrm{H}$ & -0.166769 & 4.475329 & -0.379277 \\
\hline $\mathrm{H}$ & 1.149227 & 3.296611 & -0.610483 \\
\hline $\mathrm{C}$ & 2.785221 & 2.249533 & 1.661079 \\
\hline $\mathrm{H}$ & 3.194743 & 2.619072 & 2.616545 \\
\hline $\mathrm{H}$ & 3.572473 & 2.375242 & 0.901831 \\
\hline $\mathrm{H}$ & 1.962175 & 2.922774 & 1.383988 \\
\hline $\mathrm{C}$ & 4.334024 & 0.374163 & -1.061157 \\
\hline $\mathrm{H}$ & 4.638420 & -0.683570 & -1.023180 \\
\hline $\mathrm{H}$ & 4.894700 & 0.842727 & -1.887738 \\
\hline $\mathrm{H}$ & 4.681079 & 0.842957 & -0.127026 \\
\hline $\mathrm{C}$ & 2.488835 & -2.913547 & -0.657551 \\
\hline $\mathrm{H}$ & 3.067007 & -3.816418 & -0.397378 \\
\hline $\mathrm{H}$ & 1.482566 & -3.251707 & -0.948135 \\
\hline $\mathrm{H}$ & 2.961281 & -2.475386 & -1.552258 \\
\hline
\end{tabular}

\section{Complex 4 - $\left[\mathrm{Cp}\left(\mathrm{Me}_{3} \mathrm{P}\right) \mathrm{Rh}\left(\mathrm{SiMe}_{3}\right)_{2} \mathrm{H}\right]^{+}$}

$\begin{array}{lrrr}\mathrm{Rh} & 0.004621 & -0.245585 & -0.452591 \\ \mathrm{H} & -0.073459 & -0.583916 & 1.095214 \\ \mathrm{C} & -1.223765 & -1.290745 & -2.126617\end{array}$




\begin{tabular}{|c|c|c|c|}
\hline $\mathrm{C}$ & -0.502403 & -0.208663 & -2.699649 \\
\hline $\mathrm{C}$ & 0.909829 & -0.472629 & -2.546445 \\
\hline $\mathrm{C}$ & 1.043695 & -1.728369 & -1.882380 \\
\hline C & -0.269243 & -2.218119 & -1.581673 \\
\hline $\mathrm{H}$ & -2.304831 & -1.403319 & -2.117560 \\
\hline $\mathrm{H}$ & -0.940012 & 0.645130 & -3.211148 \\
\hline $\mathrm{H}$ & 1.722107 & 0.139505 & -2.931226 \\
\hline $\mathrm{H}$ & 1.976585 & -2.240940 & -1.663128 \\
\hline $\mathrm{H}$ & -0.501173 & -3.161185 & -1.092981 \\
\hline $\mathrm{Si}$ & 2.005511 & -0.649598 & 0.992162 \\
\hline $\mathrm{Si}$ & -2.075645 & -0.528487 & 0.951498 \\
\hline $\mathrm{P}$ & 0.078590 & 2.044582 & -0.155181 \\
\hline C & -3.455632 & 0.571665 & 0.256630 \\
\hline $\mathrm{H}$ & -3.628215 & 0.422985 & -0.819565 \\
\hline $\mathrm{H}$ & -4.388414 & 0.302417 & 0.782158 \\
\hline $\mathrm{H}$ & -3.286887 & 1.644129 & 0.434011 \\
\hline C & -1.908948 & -0.172701 & 2.808469 \\
\hline $\mathrm{H}$ & -1.238660 & -0.895410 & 3.299948 \\
\hline $\mathrm{H}$ & -1.559639 & 0.838492 & 3.055277 \\
\hline $\mathrm{H}$ & -2.906781 & -0.296335 & 3.263106 \\
\hline $\mathrm{C}$ & -2.601444 & -2.343464 & 0.842264 \\
\hline $\mathrm{H}$ & -2.901679 & -2.664477 & -0.165412 \\
\hline $\mathrm{H}$ & -1.809151 & -3.018656 & 1.199783 \\
\hline $\mathrm{H}$ & -3.473137 & -2.482637 & 1.504089 \\
\hline C & 1.953869 & -2.464015 & 1.533992 \\
\hline $\mathrm{H}$ & 1.876842 & -3.162020 & 0.687588 \\
\hline $\mathrm{H}$ & 2.881913 & -2.703681 & 2.079803 \\
\hline $\mathrm{H}$ & 1.110244 & -2.665055 & 2.212394 \\
\hline C & 2.104859 & 0.400857 & 2.567008 \\
\hline $\mathrm{H}$ & 1.214882 & 0.300777 & 3.206419 \\
\hline $\mathrm{H}$ & 2.967691 & 0.036343 & 3.150310 \\
\hline $\mathrm{H}$ & 2.281190 & 1.469830 & 2.376685 \\
\hline C & 3.603354 & -0.346840 & 0.013606 \\
\hline $\mathrm{H}$ & 3.735917 & -1.044705 & -0.825381 \\
\hline $\mathrm{H}$ & 3.697082 & 0.675634 & -0.377621 \\
\hline $\mathrm{H}$ & 4.445683 & -0.509263 & 0.708681 \\
\hline C & -0.408832 & 2.808293 & 1.446961 \\
\hline $\mathrm{H}$ & -1.479826 & 2.655690 & 1.633201 \\
\hline $\mathrm{H}$ & 0.163694 & 2.376736 & 2.277099 \\
\hline $\mathrm{H}$ & -0.210168 & 3.889756 & 1.396816 \\
\hline $\mathrm{C}$ & -1.000618 & 2.942808 & -1.354680 \\
\hline $\mathrm{H}$ & -0.595674 & 2.837445 & -2.370502 \\
\hline $\mathrm{H}$ & -2.020245 & 2.537726 & -1.333339 \\
\hline $\mathrm{H}$ & -1.028814 & 4.012791 & -1.097517 \\
\hline C & 1.711517 & 2.839540 & -0.481619 \\
\hline $\mathrm{H}$ & 2.089740 & 2.534078 & -1.466683 \\
\hline $\mathrm{H}$ & 1.597359 & 3.934463 & -0.465026 \\
\hline $\mathrm{H}$ & 2.443494 & 2.547734 & 0.282401 \\
\hline
\end{tabular}

$\begin{array}{lccr}\text { Complex 5 } & -\left[\mathbf{C p}\left(\mathbf{P M e}_{3}\right) \mathbf{R h}\left(\boldsymbol{\eta}^{\mathbf{2}}-\mathbf{H}-\mathbf{S i M e}_{3}\right)(\mathbf{S i M e})\right]^{+} & \\ \mathrm{Rh} & 0.087335 & -0.215906 & -0.488657 \\ \mathrm{H} & -0.697550 & -0.163948 & 0.945809\end{array}$




\begin{tabular}{|c|c|c|c|}
\hline C & 0.805580 & -1.642559 & -2.190664 \\
\hline C & -0.599590 & -1.728250 & -2.202755 \\
\hline $\mathrm{C}$ & -1.115441 & -0.397170 & -2.441392 \\
\hline C & -0.013200 & 0.485556 & -2.648802 \\
\hline $\mathrm{C}$ & 1.188530 & -0.262188 & -2.433321 \\
\hline $\mathrm{H}$ & 1.493749 & -2.473306 & -2.043699 \\
\hline $\mathrm{H}$ & -1.189308 & -2.629268 & -2.057361 \\
\hline $\mathrm{H}$ & -2.164523 & -0.134722 & -2.560354 \\
\hline $\mathrm{H}$ & -0.076846 & 1.532324 & -2.927918 \\
\hline $\mathrm{H}$ & 2.203502 & 0.107129 & -2.563043 \\
\hline $\mathrm{Si}$ & 0.484539 & 2.109225 & 0.164565 \\
\hline $\mathrm{P}$ & 1.663990 & -1.033908 & 0.980710 \\
\hline $\mathrm{Si}$ & -2.355687 & -0.400888 & 0.787503 \\
\hline $\mathrm{C}$ & 1.310252 & -2.799407 & 1.378821 \\
\hline $\mathrm{H}$ & 1.265712 & -3.392464 & 0.455596 \\
\hline $\mathrm{H}$ & 2.093410 & -3.209022 & 2.035435 \\
\hline $\mathrm{H}$ & 0.339401 & -2.876628 & 1.888116 \\
\hline C & 1.877660 & -0.301443 & 2.652528 \\
\hline $\mathrm{H}$ & 2.230500 & 0.734705 & 2.578968 \\
\hline $\mathrm{H}$ & 0.923421 & -0.315072 & 3.196798 \\
\hline $\mathrm{H}$ & 2.616926 & -0.893397 & 3.213217 \\
\hline $\mathrm{C}$ & 3.381342 & -1.085988 & 0.319861 \\
\hline $\mathrm{H}$ & 3.414040 & -1.685150 & -0.600162 \\
\hline $\mathrm{H}$ & 3.730321 & -0.070495 & 0.092118 \\
\hline $\mathrm{H}$ & 4.052794 & -1.541021 & 1.063936 \\
\hline C & 2.330138 & 2.568534 & 0.083649 \\
\hline $\mathrm{H}$ & 2.773857 & 2.349966 & -0.899889 \\
\hline $\mathrm{H}$ & 2.417671 & 3.657233 & 0.242944 \\
\hline $\mathrm{H}$ & 2.949694 & 2.083847 & 0.853621 \\
\hline C & -0.145885 & 2.557761 & 1.904841 \\
\hline $\mathrm{H}$ & 0.205345 & 1.903969 & 2.714091 \\
\hline $\mathrm{H}$ & 0.206217 & 3.578702 & 2.132788 \\
\hline $\mathrm{H}$ & -1.245429 & 2.584683 & 1.945255 \\
\hline C & -0.402979 & 3.331379 & -0.995513 \\
\hline $\mathrm{H}$ & 0.132458 & 3.470537 & -1.947131 \\
\hline $\mathrm{H}$ & -1.442579 & 3.051740 & -1.220962 \\
\hline $\mathrm{H}$ & -0.427369 & 4.314838 & -0.495515 \\
\hline C & -2.419388 & -0.410218 & 2.679988 \\
\hline $\mathrm{H}$ & -1.862656 & -1.257777 & 3.109117 \\
\hline $\mathrm{H}$ & -2.039299 & 0.520495 & 3.124694 \\
\hline $\mathrm{H}$ & -3.473080 & -0.522460 & 2.987219 \\
\hline C & -2.994053 & -2.064932 & 0.179798 \\
\hline $\mathrm{H}$ & -3.202392 & -2.080070 & -0.898612 \\
\hline $\mathrm{H}$ & -2.307184 & -2.890026 & 0.418297 \\
\hline $\mathrm{H}$ & -3.944695 & -2.264155 & 0.703665 \\
\hline $\mathrm{C}$ & -3.354602 & 1.046684 & 0.123082 \\
\hline $\mathrm{H}$ & -3.395028 & 1.079630 & -0.974179 \\
\hline $\mathrm{H}$ & -4.388576 & 0.936441 & 0.493139 \\
\hline $\mathrm{H}$ & -2.976561 & 2.013856 & 0.483210 \\
\hline
\end{tabular}

\section{Complex 6 - $\left[\mathrm{Cp} * \mathbf{R h}\left(\mathrm{SiMe}_{3}\right)_{2}\left(\mathrm{PMe}_{3}\right) \mathrm{H}\right]^{+}$}

$\mathrm{Rh}$

0.132147
$-0.014063$

0.011740 


\begin{tabular}{|c|c|c|c|}
\hline $\mathrm{H}$ & -0.710871 & 0.280444 & -1.316333 \\
\hline C & 2.050327 & -1.337007 & -0.224978 \\
\hline C & 2.298705 & -0.034298 & -0.798062 \\
\hline $\mathrm{C}$ & 2.282368 & 0.941955 & 0.274768 \\
\hline $\mathrm{C}$ & 1.931513 & 0.258745 & 1.478484 \\
\hline C & 1.756283 & -1.157262 & 1.169432 \\
\hline C & 2.383435 & -2.633056 & -0.898967 \\
\hline $\mathrm{C}$ & 2.839417 & 0.190031 & -2.176994 \\
\hline $\mathrm{C}$ & 2.847860 & 2.327456 & 0.225806 \\
\hline $\mathrm{C}$ & 2.085536 & 0.839865 & 2.850967 \\
\hline C & 1.638810 & -2.256234 & 2.182228 \\
\hline $\mathrm{P}$ & -1.598238 & 0.054892 & 1.591725 \\
\hline $\mathrm{Si}$ & -1.120049 & -1.777482 & -1.258663 \\
\hline $\mathrm{Si}$ & -0.694871 & 2.169610 & -1.041812 \\
\hline $\mathrm{C}$ & -0.518633 & -1.783810 & -3.062722 \\
\hline $\mathrm{H}$ & 0.574487 & -1.775698 & -3.175375 \\
\hline $\mathrm{H}$ & -0.897794 & -2.687540 & -3.568724 \\
\hline $\mathrm{H}$ & -0.911859 & -0.910780 & -3.608013 \\
\hline $\mathrm{C}$ & -3.003968 & -1.552750 & -1.403291 \\
\hline $\mathrm{H}$ & -3.558008 & -1.770357 & -0.478265 \\
\hline $\mathrm{H}$ & -3.306005 & -0.562533 & -1.772688 \\
\hline $\mathrm{H}$ & -3.341051 & -2.292693 & -2.149930 \\
\hline $\mathrm{C}$ & -0.903788 & -3.530127 & -0.548092 \\
\hline $\mathrm{H}$ & 0.081719 & -3.964905 & -0.755646 \\
\hline $\mathrm{H}$ & -1.095496 & -3.608107 & 0.530750 \\
\hline $\mathrm{H}$ & -1.649836 & -4.164631 & -1.057513 \\
\hline $\mathrm{C}$ & -2.073482 & -1.572985 & 2.327829 \\
\hline $\mathrm{H}$ & -1.183634 & -2.091890 & 2.707119 \\
\hline $\mathrm{H}$ & -2.774093 & -1.409320 & 3.160809 \\
\hline $\mathrm{H}$ & -2.557275 & -2.210518 & 1.577119 \\
\hline $\mathrm{C}$ & -3.255385 & 0.764124 & 1.197659 \\
\hline $\mathrm{H}$ & -3.708755 & 0.272977 & 0.330569 \\
\hline $\mathrm{H}$ & -3.905577 & 0.616634 & 2.073386 \\
\hline $\mathrm{H}$ & -3.171467 & 1.840192 & 1.000336 \\
\hline $\mathrm{C}$ & -1.229950 & 1.005868 & 3.135909 \\
\hline $\mathrm{H}$ & -0.426827 & 0.522215 & 3.703686 \\
\hline $\mathrm{H}$ & -0.934486 & 2.038915 & 2.910566 \\
\hline $\mathrm{H}$ & -2.136045 & 1.025972 & 3.760567 \\
\hline $\mathrm{C}$ & -2.411306 & 2.165433 & -1.864793 \\
\hline $\mathrm{H}$ & -2.417575 & 1.532924 & -2.766589 \\
\hline $\mathrm{H}$ & -3.252015 & 1.861614 & -1.230498 \\
\hline $\mathrm{H}$ & -2.608508 & 3.198948 & -2.197692 \\
\hline $\mathrm{C}$ & 0.417730 & 2.718000 & -2.475675 \\
\hline $\mathrm{H}$ & 1.426599 & 3.036734 & -2.185158 \\
\hline $\mathrm{H}$ & 0.503265 & 1.939423 & -3.248338 \\
\hline $\mathrm{H}$ & -0.071846 & 3.589148 & -2.943303 \\
\hline $\mathrm{C}$ & -0.694091 & 3.529648 & 0.284097 \\
\hline $\mathrm{H}$ & 0.285379 & 3.657719 & 0.765734 \\
\hline $\mathrm{H}$ & -0.942853 & 4.480304 & -0.218576 \\
\hline $\mathrm{H}$ & -1.446474 & 3.371708 & 1.069677 \\
\hline $\mathrm{H}$ & 2.107031 & -3.498617 & -0.284966 \\
\hline $\mathrm{H}$ & 1.922490 & -2.747311 & -1.889706 \\
\hline $\mathrm{H}$ & 3.476454 & -2.675914 & -1.050162 \\
\hline
\end{tabular}




$\begin{array}{lrrr}\mathrm{H} & 3.927217 & -0.002777 & -2.178809 \\ \mathrm{H} & 2.389867 & -0.489180 & -2.913969 \\ \mathrm{H} & 2.687266 & 1.218632 & -2.524065 \\ \mathrm{H} & 2.377202 & 2.999448 & 0.956739 \\ \mathrm{H} & 3.922648 & 2.281723 & 0.477789 \\ \mathrm{H} & 2.773913 & 2.789661 & -0.765648 \\ \mathrm{H} & 1.638441 & 1.838758 & 2.944838 \\ \mathrm{H} & 1.666710 & 0.193437 & 3.632401 \\ \mathrm{H} & 3.162380 & 0.946139 & 3.071903 \\ \mathrm{H} & 1.111999 & -1.932519 & 3.090741 \\ \mathrm{H} & 1.117042 & -3.133558 & 1.777758 \\ \mathrm{H} & 2.643795 & -2.586713 & 2.498305\end{array}$

$\begin{array}{lccc}\text { Complex } & \text { - }[\mathbf{C p} \text { *Rh(SiMe })\left(\mathbf{H ~ S i M e}_{3}\right) & \left.\left(\mathbf{P M e}_{3}\right)\right]^{+} \\ \mathrm{Rh} & 0.2808 & 0.05584 & -0.01983 \\ \mathrm{H} & -1.46001 & 0.2522 & -0.03701 \\ \mathrm{C} & 2.00815 & 1.24141 & -1.08791 \\ \mathrm{C} & 1.1542 & 2.21468 & -0.52794 \\ \mathrm{C} & 1.07218 & 1.98125 & 0.92211 \\ \mathrm{C} & 1.96003 & 0.90874 & 1.2494 \\ \mathrm{C} & 2.46464 & 0.36674 & 0.0012 \\ \mathrm{C} & 2.52336 & 1.22523 & -2.49371 \\ \mathrm{C} & 0.59089 & 3.40262 & -1.24283 \\ \mathrm{C} & 0.41917 & 2.91337 & 1.89672 \\ \mathrm{C} & 2.53305 & 0.5834 & 2.59348 \\ \mathrm{C} & 3.60713 & -0.59875 & -0.07381 \\ \mathrm{Si} & -0.32971 & -1.46864 & 1.78866 \\ \mathrm{P} & 0.06307 & -1.61633 & -1.61951 \\ \mathrm{Si} & -2.87686 & 0.95597 & -0.26535 \\ \mathrm{C} & -0.38359 & -0.91323 & -3.27297 \\ \mathrm{H} & 0.30887 & -0.10649 & -3.54583 \\ \mathrm{H} & -0.35224 & -1.69495 & -4.04778 \\ \mathrm{H} & -1.40065 & -0.49842 & -3.23042 \\ \mathrm{C} & -1.16483 & -2.98897 & -1.50351 \\ \mathrm{H} & -0.90953 & -3.66309 & -0.67595 \\ \mathrm{H} & -2.1759 & -2.59532 & -1.34437 \\ \mathrm{H} & -1.14513 & -3.55885 & -2.44504 \\ \mathrm{C} & 1.58596 & -2.58735 & -2.00102 \\ \mathrm{H} & 2.36533 & -1.9381 & -2.41839 \\ \mathrm{H} & 1.97019 & -3.05613 & -1.08477 \\ \mathrm{H} & 1.34911 & -3.36963 & -2.7377 \\ \mathrm{C} & 0.8963 & -2.91822 & 1.98065 \\ \mathrm{H} & 1.94087 & -2.59492 & 2.11083 \\ \mathrm{H} & 0.62539 & -3.50383 & 2.87588 \\ \mathrm{H} & 0.86707 & -3.60826 & 1.12201 \\ \mathrm{C} & -2.06876 & -2.2597 & 1.7136 \\ \mathrm{H} & -2.31052 & -2.84528 & 0.82005 \\ \mathrm{H} & -2.13801 & -2.94251 & 2.57826 \\ \mathrm{H} & -2.854 & -1.50051 & 1.85138 \\ \mathrm{C} & -0.44428 & -0.56354 & 3.46962 \\ \mathrm{H} & 0.51951 & -0.44606 & 3.97973 \\ \mathrm{H} & -0.91161 & 0.42824 & 3.37474 \\ & & & \end{array}$




$\begin{array}{lrrr}\mathrm{H} & -1.08931 & -1.16576 & 4.13119 \\ \mathrm{C} & -3.98871 & -0.42139 & -0.90081 \\ \mathrm{H} & -3.69928 & -0.75901 & -1.90731 \\ \mathrm{H} & -4.02319 & -1.28907 & -0.22825 \\ \mathrm{H} & -5.01314 & -0.01848 & -0.97746 \\ \mathrm{C} & -2.76963 & 2.32745 & -1.5427 \\ \mathrm{H} & -2.27863 & 3.23192 & -1.1609 \\ \mathrm{H} & -2.25241 & 2.00909 & -2.45962 \\ \mathrm{H} & -3.79962 & 2.60541 & -1.82399 \\ \mathrm{C} & -3.38915 & 1.57942 & 1.42888 \\ \mathrm{H} & -2.69039 & 2.3322 & 1.82129 \\ \mathrm{H} & -4.38132 & 2.05436 & 1.34839 \\ \mathrm{H} & -3.46569 & 0.76512 & 2.16375 \\ \mathrm{H} & 2.71628 & 0.20945 & -2.86605 \\ \mathrm{H} & 1.83501 & 1.72316 & -3.19127 \\ \mathrm{H} & 3.48389 & 1.76762 & -2.54482 \\ \mathrm{H} & -0.24267 & 3.85959 & -0.69434 \\ \mathrm{H} & 1.37104 & 4.17842 & -1.33915 \\ \mathrm{H} & 0.24602 & 3.16139 & -2.25813 \\ \mathrm{H} & 0.19424 & 2.41904 & 2.85103 \\ \mathrm{H} & 1.08822 & 3.76483 & 2.11352 \\ \mathrm{H} & -0.51632 & 3.33538 & 1.50232 \\ \mathrm{H} & 2.58317 & -0.49663 & 2.78815 \\ \mathrm{H} & 3.56686 & 0.96971 & 2.63667 \\ \mathrm{H} & 1.97159 & 1.05663 & 3.40758 \\ \mathrm{H} & 3.78841 & -0.96315 & -1.09204 \\ \mathrm{H} & 4.53182 & -0.09253 & 0.25714 \\ \mathrm{H} & 3.45989 & -1.46593 & 0.58477\end{array}$

\begin{tabular}{lrrr}
\multicolumn{4}{l}{ Complex $8-\left[\mathbf{C p R h}\left(\mathbf{S i M e}_{3}\right)(\mathbf{H})_{\mathbf{2}}\left(\mathbf{P M e}_{3}\right)\right]^{+}$} \\
$\mathrm{Rh}$ & -0.049732 & -0.400382 & \\
$\mathrm{H}$ & 0.308925 & 0.639654 & -0.028407 \\
$\mathrm{C}$ & 1.236865 & -2.255564 & 0.110725 \\
$\mathrm{C}$ & 0.244962 & -2.302704 & 1.224275 \\
$\mathrm{C}$ & -1.034219 & -2.368357 & 0.603859 \\
$\mathrm{C}$ & -0.843313 & -2.374393 & -0.829666 \\
$\mathrm{C}$ & 0.559866 & -2.316322 & -1.081628 \\
$\mathrm{H}$ & 2.314000 & -2.243125 & 0.330902 \\
$\mathrm{H}$ & 0.440296 & -2.295349 & 2.294250 \\
$\mathrm{H}$ & -1.988324 & -2.443451 & 1.122278 \\
$\mathrm{H}$ & -1.622050 & -2.464521 & -1.583235 \\
$\mathrm{H}$ & 1.034864 & -2.319446 & -2.060186 \\
$\mathrm{H}$ & 0.250079 & 0.546961 & -1.250689 \\
$\mathrm{Si}$ & 2.058584 & 0.965070 & 0.003316 \\
$\mathrm{C}$ & 2.988797 & 0.616055 & 1.613783 \\
$\mathrm{H}$ & 3.187340 & -0.453578 & 1.774583 \\
$\mathrm{H}$ & 3.963436 & 1.131508 & 1.572005 \\
$\mathrm{H}$ & 2.448114 & 0.996764 & 2.493441 \\
$\mathrm{C}$ & 1.775447 & 2.825910 & -0.129202 \\
$\mathrm{H}$ & 1.255530 & 3.117032 & -1.053488 \\
$\mathrm{H}$ & 1.224169 & 3.232847 & 0.732272 \\
$\mathrm{H}$ & 2.762835 & 3.317719 & -0.141247
\end{tabular}




$\begin{array}{lr}\mathrm{C} & 3.099632 \\ \mathrm{H} & 3.359495 \\ \mathrm{H} & 2.602314 \\ \mathrm{H} & 4.045179 \\ \mathrm{P} & -1.948005 \\ \mathrm{C} & -1.772960 \\ \mathrm{H} & -1.039527 \\ \mathrm{H} & -1.434003 \\ \mathrm{H} & -2.745928 \\ \mathrm{C} & -2.715936 \\ \mathrm{H} & -2.922847 \\ \mathrm{H} & -2.025176 \\ \mathrm{H} & -3.655922 \\ \mathrm{C} & -3.282813 \\ \mathrm{H} & -3.589807 \\ \mathrm{H} & -4.154542 \\ \mathrm{H} & -2.918606\end{array}$

$\begin{array}{rr}0.398692 & -1.471648 \\ -0.669453 & -1.431222 \\ 0.596141 & -2.432779 \\ 0.967582 & -1.460515 \\ 0.904179 & 0.002897 \\ 2.643537 & -0.542864 \\ 3.166546 & 0.083498 \\ 2.675862 & -1.587261 \\ 3.151610 & -0.462798 \\ 1.027959 & 1.666473 \\ 0.022409 & 2.056931 \\ 1.532743 & 2.355836 \\ 1.597908 & 1.611104 \\ 0.241428 & -1.072112 \\ -0.755046 & -0.726231 \\ 0.913078 & -1.044126 \\ 0.163715 & -2.105560\end{array}$

\section{Transition state TS}

$\begin{array}{lrrr}\mathrm{Rh} & -0.062917 & -0.028584 & 0.472774 \\ \mathrm{H} & -0.207550 & 0.039396 & -1.089163 \\ \mathrm{C} & 0.356937 & 0.861562 & 2.576006 \\ \mathrm{C} & -1.064033 & 0.860990 & 2.441332 \\ \mathrm{C} & -1.486229 & -0.506746 & 2.341129 \\ \mathrm{C} & -0.341221 & -1.345266 & 2.421570 \\ \mathrm{C} & 0.813600 & -0.497426 & 2.548078 \\ \mathrm{H} & 0.985231 & 1.737544 & 2.710158 \\ \mathrm{H} & -1.710000 & 1.734937 & 2.440885 \\ \mathrm{H} & -2.514276 & -0.849390 & 2.246657 \\ \mathrm{H} & -0.340569 & -2.430998 & 2.393655 \\ \mathrm{H} & 1.841749 & -0.827146 & 2.677623 \\ \mathrm{Si} & 1.405024 & -1.611447 & -0.591624 \\ \mathrm{Si} & 0.979499 & 1.914541 & -0.489120 \\ \mathrm{Si} & -2.103861 & -0.201174 & -0.878084 \\ \mathrm{C} & -0.222541 & 3.392040 & -0.363837 \\ \mathrm{H} & -0.570899 & 3.551139 & 0.668193 \\ \mathrm{H} & 0.285160 & 4.314412 & -0.696371 \\ \mathrm{H} & -1.110219 & 3.253227 & -1.000673 \\ \mathrm{C} & 1.475593 & 1.819633 & -2.329344 \\ \mathrm{H} & 2.264049 & 1.074410 & -2.514861 \\ \mathrm{H} & 0.624512 & 1.580590 & -2.985215 \\ \mathrm{H} & 1.870392 & 2.802245 & -2.640343 \\ \mathrm{C} & 2.572560 & 2.450755 & 0.420976 \\ \mathrm{H} & 2.360152 & 2.876191 & 1.413727 \\ \mathrm{H} & 3.290378 & 1.627735 & 0.550322 \\ \mathrm{H} & 3.067878 & 3.237788 & -0.173976 \\ \mathrm{C} & 3.252179 & -1.153925 & -0.442666 \\ \mathrm{H} & 3.553214 & -1.000950 & 0.605488 \\ \mathrm{H} & 3.861765 & -1.979390 & -0.850252 \\ \mathrm{H} & 3.508566 & -0.241926 & -1.001972 \\ \mathrm{C} & 1.090555 & -1.943975 & -2.444690 \\ \mathrm{H} & 1.184638 & -1.039563 & -3.064537\end{array}$




$\begin{array}{lrrr}\mathrm{H} & 1.828397 & -2.678667 & -2.810420 \\ \mathrm{H} & 0.089291 & -2.370322 & -2.616345 \\ \mathrm{C} & 1.249136 & -3.329906 & 0.230808 \\ \mathrm{H} & 1.587008 & -3.321026 & 1.278557 \\ \mathrm{H} & 0.215168 & -3.706944 & 0.206364 \\ \mathrm{H} & 1.883244 & -4.047984 & -0.317986 \\ \mathrm{C} & -2.065813 & 0.262268 & -2.727588 \\ \mathrm{H} & -1.805587 & 1.321912 & -2.877683 \\ \mathrm{H} & -1.358642 & -0.348124 & -3.309004 \\ \mathrm{H} & -3.072861 & 0.105963 & -3.150598 \\ \mathrm{C} & -3.422330 & 0.956257 & -0.138372 \\ \mathrm{H} & -3.724989 & 0.667620 & 0.878422 \\ \mathrm{H} & -3.066589 & 1.996874 & -0.104604 \\ \mathrm{H} & -4.319082 & 0.926433 & -0.781321 \\ \mathrm{C} & -2.716949 & -1.998511 & -0.795258 \\ \mathrm{H} & -2.856639 & -2.344189 & 0.239692 \\ \mathrm{H} & -3.683444 & -2.086030 & -1.321277 \\ \mathrm{H} & -2.006111 & -2.682881 & -1.283220\end{array}$

